09.02 - Physiotherapists

\title{
12583
}

\section{Relationship between family function and exercise capacity and inspiratory muscle strength in COPD}

COPD, Rehabilitation, Chronic diseases

C. F. Pinto da Paixão ${ }^{1}$, P. Rebelo ${ }^{1}$, A. Oliveira ${ }^{1}$, C. Jácome ${ }^{2}$, J. Cruz $^{3}$, C. Freitas ${ }^{4}$, H. Loureiro $^{5}$, M. Rua ${ }^{4}$, P. Ferreira ${ }^{5}$, A. Marques ${ }^{5}$

${ }^{1}$ Lab3R - Respiratory Research and Rehabilitation Laboratory, School of Health Sciences, University of Aveiro (ESSUA) - Aveiro (Portugal), ${ }^{2}$ CINTESIS - Center for Health Technology and Services Research - Porto (Portugal), ${ }^{3}$ Health Research Unit (UIS), School of Health Sciences, Polytechnic Institute of Leiria (ESSLei) - Leiria (Portugal), ${ }^{4}$ Research Centre on Didactics and Technology in the Education of Trainers - CIDTFF - Aveiro (Portugal), ${ }^{5}$ Institute for Research in Biomedicine (iBiMED) Aveiro (Portugal)

Chronic obstructive pulmonary disease (COPD) is a multidimensional disease known to affect not only patients but also family functioning. Family functioning affects several dimensions of patients' psychological and social performance, however the association with patient's physical outcomes remains unknown. This study explored associations between family function and exercise capacity and inspiratory muscle strength in COPD.

20 patients with COPD ( $85 \%$ male; $67.5 \pm 10 y$; $54.3 \pm 29.4 \mathrm{FEV} 1 \%$ predicted) were included.

Sociodemographic, anthropometric and lung function data were collected to characterise the sample. 2 sixminute walk tests (6MWT) and up to 5 maximum inspiratory pressure (MIP) manoeuvres were performed. The best performances were analysed. Family function was assessed with the family adaptability and cohesion evaluation scale (FACES-IV). Correlations were explored with the Spearman's correlation coefficient.

Moderate and positive correlations were found between family satisfaction and 6MWT $(r=0.55 ; p=0.02)$ and between family cohesion and MIP values $(r=0.59 ; p=0.01)$. Strong and positive correlations $(r=0.61 ; p=0.01)$ were found between family satisfaction and MIP values. No further correlations were found (Fig.1). 
Family function correlated significantly with patients' exercise capacity and inspiratory muscle strength.

Further research on family functioning is needed to enhance knowledge on COPD management.
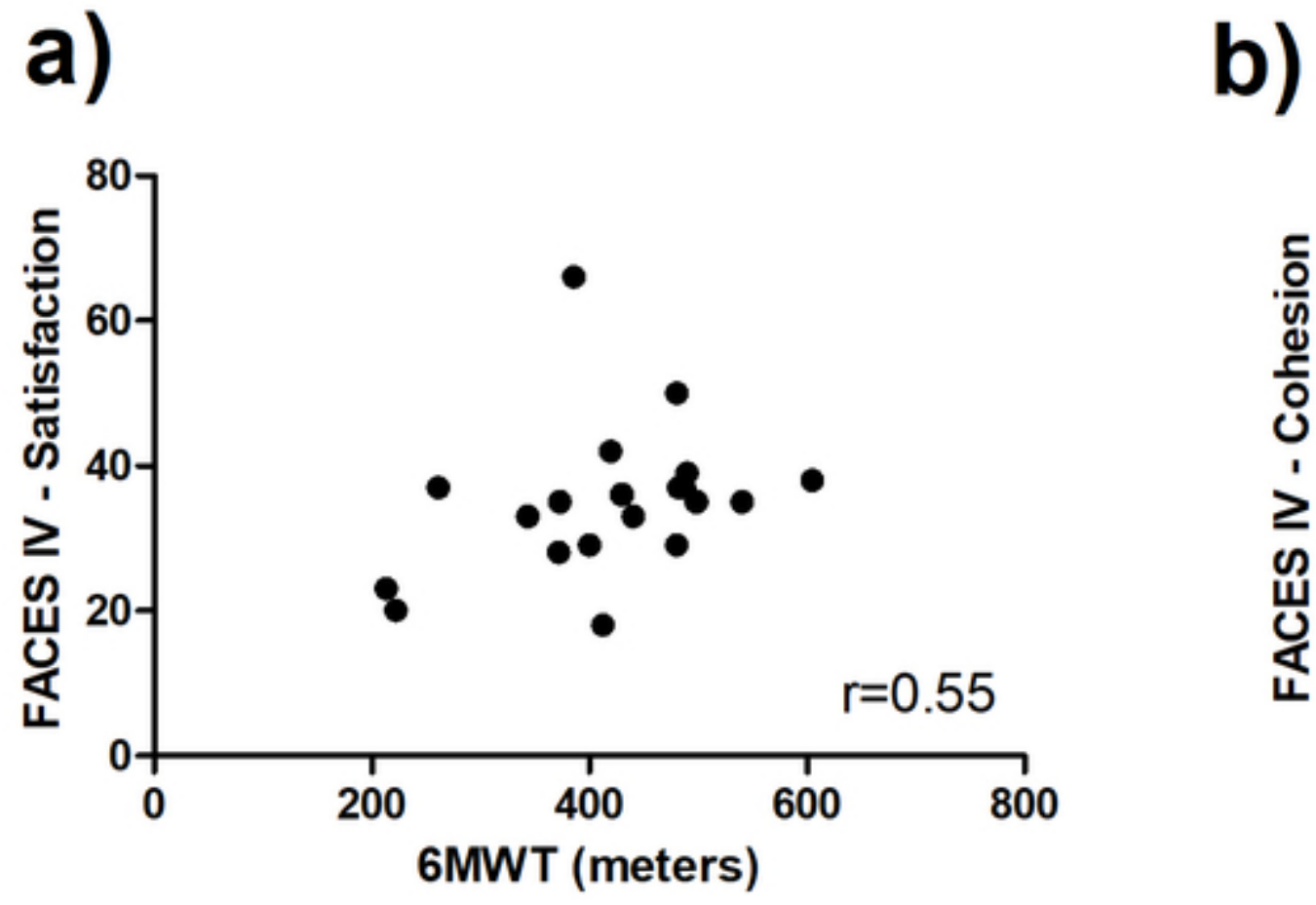

c)

d)
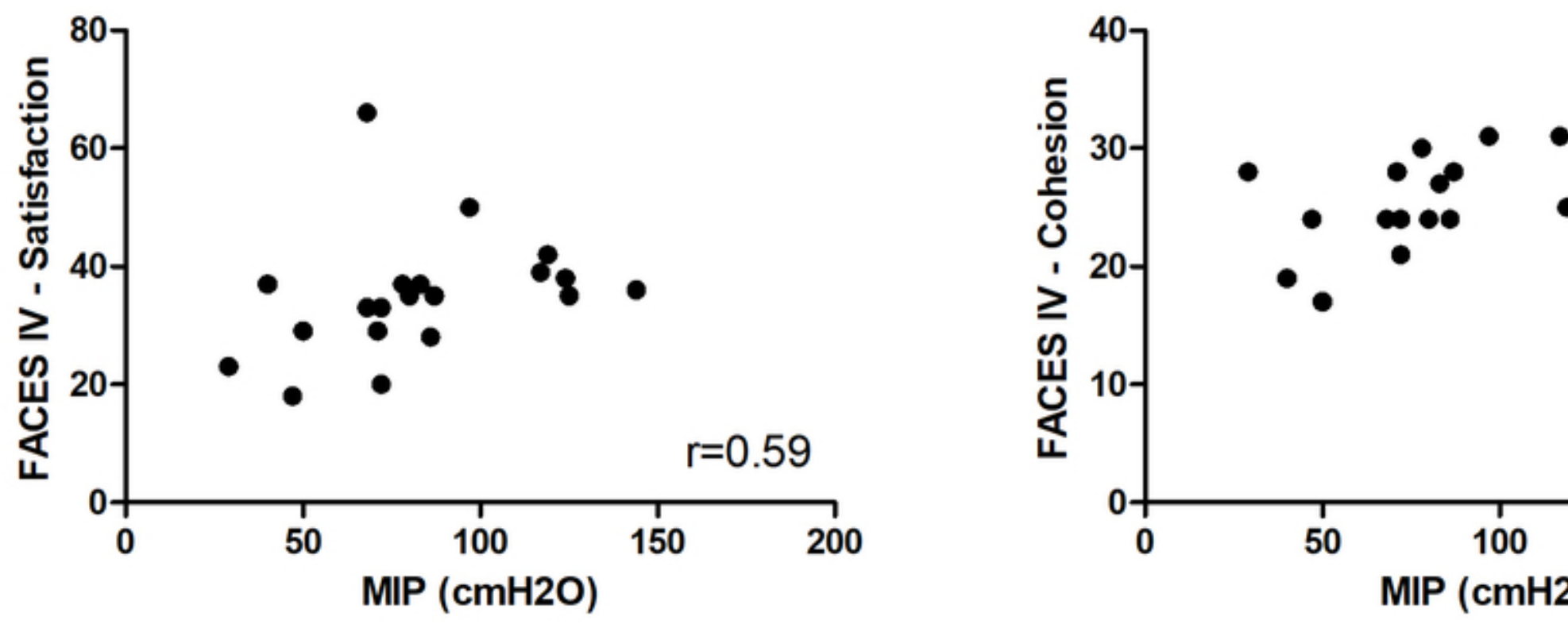

Fig. 1 - Correlations between: a) 6-min. walk test (6MWT) and family satisfaction; b) $6 \mathrm{MW}$ c) maximum inspiratory pressure (MIP) and family satisfaction and d) MIP and family cohe 
